\title{
Commentary on key aspects of fecal microbiota transplantation in small animal practice
}

This article was published in the following Dove Press journal:

Veterinary Medicine: Research and Reports

31 May 2016

Number of times this article has been viewed

\author{
Jennifer Chaitman' \\ Albert E Jergens ${ }^{2}$ \\ Frederic Gaschen ${ }^{3}$ \\ Jose F Garcia-Mazcorro ${ }^{4}$ \\ Stanley L Marks ${ }^{5}$ \\ Alicia G Marroquin- \\ Cardona ${ }^{4}$ \\ Keith Richter ${ }^{6}$ \\ Giacomo Rossi ${ }^{7}$ \\ Jan S Suchodolski ${ }^{8}$ \\ J Scott Weese? \\ 'Veterinary Internal Medicine and \\ Allergy Specialists, New York, NY, \\ ${ }^{2}$ College of Veterinary Medicine, \\ lowa State University, Ames, IA, \\ ${ }^{3}$ School of Veterinary Medicine, \\ Lousiana State University, LA, USA; \\ ${ }^{4}$ Faculty of Veterinary Medicine, \\ Universidad Autónoma de Nuevo \\ León, General Escobedo, Nuevo León \\ Mexico; ${ }^{5}$ Department of Medicine \& \\ Epidemiology, School of Veterinary \\ Medicine, University of California, \\ Davis, Davis, ${ }^{6}$ Veterinary Specialty \\ Hospital of San Diego, San Diego, \\ CA, USA; ' Department of Veterinary \\ Science, School of Veterinary Medical \\ Sciences, University of Camerino, \\ Camerino, Italy; ${ }^{8} \mathrm{G}$ astrointestinal \\ Laboratory, Texas A\&M University, \\ College Station, TX, USA; ' Ontario \\ Veterinary College, University of \\ Guelph, Guelph, ON, Canada
}

Correspondence: Jose F Garcia-Mazcorro Faculty of Veterinary Medicine, Universidad Autónoma de Nuevo Leon, Francisco Villa s/n Colonia Ex-Hacienda el Canada, General Escobedo, Nuevo Leon, CP 66050, Mexico

$\mathrm{Tel}+52$ I 3404390 ext3624

Email jose.garciamzc@uanl.edu.mx

\begin{abstract}
The gastrointestinal tract of dogs, cats, and other mammals including humans harbors millions of beneficial microorganisms that regulate and maintain health. Fecal microbiota transplantation (FMT) is a procedure involving the administration of a fecal infusion from a healthy individual (donor) to a patient with disease to help improve health. Despite the effectiveness of FMT to treat intestinal disorders in humans, in particular recurrent Clostridium difficile infection, there is a paucity of scientific data regarding the application of FMT in veterinary patients. Here, we outline key aspects of FMT in small animal practice.
\end{abstract}

Keywords: microbiota, health, fecal microbiota transplantation, dysbiosis, enteropathogens, immune system

\section{Fecal microbiota transplantation in small animal practice}

For many years, veterinarians have used the transference of microbial communities from healthy animals to sick animals in an effort to improve health, especially in ruminants. ${ }^{1}$ Fecal microbiota transplantation (FMT) is a procedure involving the administration of a fecal infusion from a healthy individual (donor) to a patient with disease. In human medicine, FMT has been used successfully to treat recurrent Clostridium difficile infection $(\mathrm{CDI})^{2}$ and may be indicated for other gastrointestinal (GI) and non-GI disorders. ${ }^{3,4}$ FMT use in humans for treatment of disease, particularly chronic intestinal disorders, has been comprehensively reviewed. ${ }^{2}$ In sharp contrast, there are no published peerreviewed studies with regard to the use of FMT in dogs and cats. During the annual meeting of the Comparative Gastroenterology Association in Placencia, Belize (March 7-11, 2015), a group of veterinarians with diverse interests in gastroenterology raised this concern and decided to integrate a team of experts to provide insight about FMT in small animal medicine to the veterinary community worldwide.

\section{Mechanism}

Dietary supplements such as probiotics, prebiotics, and their combination (ie, synbiotics) have the potential of improving health in small animals through a modification of the intestinal microbiota. ${ }^{5}$ The rationale of using these products is similar to the rationale of using FMT in human and veterinary medicine: to help improve health in sick patients. However, the potential benefit of probiotic products in some GI disorders, including idiopathic inflammatory bowel disease (IBD), is arguably modest at best for a subset of patients. The mechanisms by which FMT confers health benefits to patients 
are not well understood but appear to be related to a restoration or restructuring of the gut microbiota. This hypothesis is supported by evidence on the beneficial effects that gut microorganisms exert on health and also by the observation, in humans, that an FMT recipient can adopt and maintain the transplanted microbiota. ${ }^{6}$ It is also possible that FMT acts as a form of immunotherapy with multiple host-microbiota mechanisms likely contributing to improved gut homeostasis and host health.

\section{Indications}

Currently, there is a lack of guidelines to perform FMT in small animals. In practice, veterinarians may consider using FMT when there are no other options to treat a particular GI disorder in a patient. Current recommendations in humans focus on the treatment of recurrent $\mathrm{CDI},{ }^{2}$ a disease that is of lesser clinical relevance in small animals. ${ }^{7}$ In dogs and cats, FMT may have the potential to improve health in any disease associated with an alteration or dysbiosis of intestinal microbial ecology such as acute and chronic GI inflammation ${ }^{7,8}$ and idiopathic diarrhea as well as IBD. ${ }^{9-12}$ Importantly, dysbiosis refers to an alteration in normal microbial composition and is by itself a poorly understood phenomenon that is currently best assessed by using molecular approaches to describe the diverse bacterial taxa. For example, a significant association between diarrhea and Clostridium perfringens enterotoxin or toxin A for both $C$. perfringens and $C$. difficile has been described in dogs, ${ }^{13}$ while other studies reported that dogs with diarrhea show a dysbiosis that is irrespective of the presence of $C$. perfringens and $C$. perfringens enterotoxin. ${ }^{14}$ In addition, clinically healthy dogs harbor vastly different amounts of microorganisms, thus challenging the concept of a "normal" gut microbial state common to all healthy individuals. ${ }^{15}$ It is also important to emphasize that in human medicine good data exist only for recurrent $C$. difficile and that the use of FMT for other disorders such as IBD is not yet well supported by scientific evidence.

\section{Donor selection}

The optimal donor characteristics are unknown. Guidance from humans is relatively limited because of the lack of a standardized approach and limited data comparing methods used by different clinicians. Both relatives and nonrelatives have been used in humans, where selection is based on specific exclusion criteria derived from the medical history and baseline laboratory testing (in particular, serum/fecal pathogen recognition). Importantly, our full understanding of the mechanisms by which enteropathogens cause diarrhea in dogs and cats is limited. ${ }^{7}$ In dogs, large and small breeds can be donors as long as they produce sufficient feces for the transplant. Animals that are in poor health, obese, and nonvaccinated against relevant infectious diseases, demonstrate clinical signs or have a history of GI disease, have a history of recent (past 3 months) antibiotic use, are atopic or food allergic, and/or are diagnosed or suspected of being infected with an enteropathogen, including bacteria, viruses, fungi, and parasites (both endo- and ectoparasites), should be excluded as potential fecal donors. Different clinicians opt for different screening procedures, and at this point, we cannot recommend any specific serological or stool testing protocol.

\section{Preparation}

Different techniques for preparing the donor fecal infusion for transplantation have been described. One human protocol calls for mixing the feces ( $\sim 50 \mathrm{~g})$ with normal sterile saline $(\sim 250 \mathrm{~mL}) .{ }^{16}$ The feces are then thoroughly homogenized and sometimes filtered to remove particulates, although it is unknown whether the effect of filtration improves clinical efficacy in humans. Filtering the feces through a tea strainer or cheesecloth facilitates the removal of hair and other particulate matter that will reduce the likelihood of the fecal homogenate clogging the tube or biopsy channel of an endoscope if this instrument is used for the FMT procedure. Fresh as well as previously frozen transplants have proved clinical efficacy in humans, ${ }^{17}$ the use of glycerol (final concentration $10 \%$ ) as cryoprotectant is common to preserve the viability of the microbiota during freezing, although this protective effect may vary among different microorganisms. Various volumes ( $300-700 \mathrm{~mL})$ of fecal infusions have been used for individual transplants in people. In human studies, capsules containing donor feces have been successfully used to deliver the desired microbiota per os. ${ }^{18}$ This could possibly serve as an alternative route of administration of interest for veterinary clinicians.

\section{Administration}

FMT may be performed either orally (eg, nasoduodenal intubation and enteroscopy) or rectally (ie, rectal enema and colonoscopy). Oral FMT may be easier to perform, but several hours are needed for the transplant to reach the large intestine, and survival of organisms throughout the stomach and small intestine may be of concern. More severe cases and those patients having clinical signs of distal small intestinal or colonic involvement may require more direct rectal administration. A recent meta-analysis of 14 studies involving 305 human patients documented FMT delivered via the lower GI 
tract to be more effective for the prevention of recurrence of CDI compared to FMT delivered via the upper GI route. ${ }^{19}$ Currently, we cannot suggest one route over the other due to the absence of evidence-based data in the veterinary literature. The exact delivery location in each patient will vary depending on the size of the patient, although a colonoscope will facilitate the administration of the fecal suspension in the ascending colon, ileum, and cecum, and can be used to procure colonic biopsies before the FMT procedure. The infused fecal material should remain in the intestinal tract for as long as possible to enhance adequate mucosal contact and "engraftment" of the donor microbiota. Sedation is often enough to perform FMT unless the patient is also undergoing colonoscopy. Drugs that slow intestinal motility may be helpful to increase the time of the infusion inside the digestive tract but they are not recommended if the patient has an infectious cause for diarrhea. The need for pre-FMT enemas in the recipient to ensure removal of retained feces and enhance the success of the FMT procedure is controversial. FMT has been used in combination with drugs affecting the immune system with promising results. ${ }^{20}$

\section{Safety}

Besides lack of data regarding treatment guidelines and methods of FMT, there are only limited data in a few cases on the clinical efficacy of FMT in small animals. ${ }^{21,22}$ It is therefore impossible to determine the safety of the procedure, although adverse effects are uncommon in humans. ${ }^{2,23}$ Potential adverse effects might include direct pathogen transmission of infectious agents or weight gain; ${ }^{24}$ however, rigid screening guidelines for donors might exclude them in the first place.

\section{Regulation}

Regulation of FMT in humans varies widely among countries. Although there are currently no specific regulations regarding FMT use in veterinary medicine in the US or other countries, the US Food and Drug Administration states that "If fecal microbiota is intended for use to treat/prevent disease in and/or affect the structure or function of the body of animals, it would be considered as a drug under section 201(g) [21 USC 321(g)] of the Federal Food, Drug, and Cosmetic Act (the Act) and a new animal drug under section 201(v) [21 USC 321(v)] of the Act. New animal drugs may not be legally marketed in the US in the absence of an approved new animal drug application, abbreviated new animal drug application, conditional approval, or an index listing, under sections 512 [21 USC 360(b)], 571 [21 USC 360(c)(c)(c)], and 572 [21 USC 360(c)(c)(c)-1], of the Act. At this time, Center of Veterinary Medicine has not developed any specific policy of enforcement discretion regarding investigational new animal drug requirements for use of fecal microbiota for transplantation to treat CDI. But firms may reach out to the Center of Veterinary Medicine's Office of New Animal Drug Evaluation if they are interested in further discussion about the investigational requirements for this use" (Regulatory Counsel of the Office of Surveillance and Compliance, Center for Veterinary Medicine/Food and Drug Administration, personal communication, 2016). In the absence of objective criteria, well-designed veterinary clinical trials are required to establish best practice guidelines and more robust safety data than currently exist.

\section{Conclusion}

There is little doubt about the potential advantages of FMT to restore GI health in humans with select diseases. However, there is currently very limited scientific data in veterinary patients. This communication represents an initial attempt to update and educate the veterinary community concerning FMT in clinical practice. With emerging clinical trial experiences and effective communication, practical guidelines and recommendations can be made (albeit cautiously as in human medicine) using more objective scientific evidence. Ongoing clinical and basic science research studies will bring the strength of science to clinical observation and enhance our understanding of how important the gut microbiota is to host health.

\section{Author contributions}

All authors contributed toward data analysis, drafting and critically revising the paper and agree to be accountable for all aspects of the work.

\section{Disclosure}

AEJ, FG, JFGM, SLM, KR, GR, JSS, and JSW are members of the Comparative Gastroenterology Society (http://vetmed. tamu.edu/cgs). AGMC is a member of the American Academy of Veterinary Pharmacology and Therapeutics (https:// aavpt.site-ym.com/). The authors report no other conflicts of interest in this work.

\section{References}

1. Brag S, Hansen HJ. Treatment of ruminal indigestion according to popular belief in Sweden. Rev Sci Tech. 1994;13(2):529-535.

2. Kelly CR, Kahn S, Kashyap P, et al. Update on fecal microbiota transplantation 2015: indications, methodologies, mechanisms, and outlook. Gastroenterology. 2015;149(1):223-237. 
3. Anderson JL, Edney RJ, Whelan K. Systematic review: faecal microbiota transplantation in the management of inflammatory bowel disease. Aliment Pharmacol Ther. 2012;36(6):503-516.

4. Sha S, Liang J, Chen M, et al. Systematic review: faecal microbiota transplantation therapy for digestive and non-digestive disorders in adults and children. Aliment Pharmacol Ther. 2014;39(10): 1003-1032.

5. Schmitz S, Suchodolski JS. Understanding the canine intestinal microbiota and its modification by pro-, pre- and synbiotics - what is the evidence? Vet Med Sci. In press 2016.

6. Hamilton MJ, Weingarden AR, Unno T, Khoruts A, Sadowsky MJ. High-throughput DNA sequence analysis reveals stable engraftment of gut microbiota following transplantation of previously frozen fecal bacteria. Gut Microbes. 2013;4(2):125-135.

7. Marks SL, Rankin SC, Byrne BA, Weese JS. Enteropathogenic bacteria in dogs and cats: diagnosis, epidemiology, treatment, and control. JVet Intern Med. 2011;25(6):1195-1208.

8. Honneffer JB, Minamoto Y, Suchodolski JS. Microbiota alterations in acute and chronic gastrointestinal inflammation of cats and dogs. World J Gastroenterol. 2014;20(44):16489-16497.

9. Minamoto Y, Otoni CC, Steelman SM, et al. Alteration of the fecal microbiota and serum metabolite profiles in dogs with idiopathic inflammatory bowel disease. Gut Microbes. 2015;6(1):33-47.

10. Suchodolski JS, Markel ME, Garcia-Mazcorro JF, et al. The fecal microbiome in dogs with acute diarrhea and idiopathic inflammatory bowel disease. PLoS One. 2012;7(12):e51907.

11. Suchodolski JS, Foster ML, Sohail MU, et al. The fecal microbiome in cats with diarrhea. PLoS One. 2015;10(5):e0127378.

12. Guard BC, Barr JW, Reddivari L, et al. Characterization of microbial dysbiosis and metabolomics changes in dogs with acute diarrhea. PLoS One. 2015;10(5):e0127259.

13. Marks SL, Kather EJ, Kass PH, Melli AC. Genotypic and phenotypic characterization of Clostridium perfringens and Clostridium difficile in diarrheic and healthy dogs. J Vet Intern Med. 2002;16(5): 533-540.
14. Minamoto Y, Dhanani N, Markel ME, Steiner JM, Suchodolski JS. Prevalence of Clostridium perfringens, Clostridium perfringens enterotoxin and dysbiosis in fecal samples of dogs with diarrhea. Vet Microbiol. 2014;174(3-4):463-473.

15. Garcia-Mazcorro JF, Dowd SE, Poulsen J, Steiner JM, Suchodolski JS. Abundance and short-term temporal variability of fecal microbiota in healthy dogs. Microbiologyopen. 2012;1(3):340-347.

16. Hamilton MJ, Weingarden AR, Sadowsky MJ, Khourts A. Standardized frozen preparation for transplantation of fecal microbiota for recurrent Clostridium difficile infection. Am J Gastroenterol. 2012;107(5):761-767.

17. Brandt LJ, Aroniadis OC. An overview of fecal microbiota transplantation: techniques, indications, and outcomes. Gastroenterol Endosc. 2013;78(2):240-249.

18. Youngster I, Russell GH, Pindar C, Ziv-Baran T, Sauk J, Hohmann EL. Oral, capsulized, frozen fecal microbiota transplantation for relapsing Clostridium difficile infection. JAMA. 2014;312(17):1772-1778.

19. Furuya-Kanamori L, Doi SA, Paterson DL, et al. Upper versus lower gastrointestinal delivery for transplantation of fecal microbiota in recurrence or refractory Clostridium difficile infection: a collaborative analysis of individual patient data from 14 studies. J Clin Gastroenterol. In press 2016.

20. Cui B, Li P, Xu L, et al. Step-up fecal microbiota transplantation (FMT) strategy. Gut Microbes. 2016;3:1-6.

21. Murphy T, Chaitman J, Han E. Use of fecal transplant in eight dogs with refractory Clostridium perfringens associated diarrhea [abstract]. JVet Intern Med. 2014;28:976-1134.

22. Weese JS, Costa MC, Webb JA. Preliminary clinical and microbiome assessment of stool transplantation in the dog and the cat [abstract]. J Vet Intern Med. 2013;27(3):604-756.

23. Kelly CR, Ihynnah C, Fischer M, et al. Fecal microbiota transplant for treatment of Clostridium difficile infection in immunocompromised patients. Am J Gastroenterol. 2014;109(7):1065-1071.

24. Alang N, Kelly CR. Weight gain after fecal microbiota transplantation. Open Forum Infect Dis. 2015;2(1):1-2.
Veterinary Medicine: Research and Reports

\section{Publish your work in this journal}

Veterinary Medicine: Research and Reports is an international, peer-reviewed, open access journal publishing original research, case reports, editorials, reviews and commentaries on all areas of veterinary medicine. The manuscript management system is completely online and includes a very quick and fair peer-review system.

\section{Dovepress}

Visit http://www.dovepress.com/testimonials.php to read real quotes from published authors. 